\title{
Contribuição dos insumos no custo total do bioprocesso para produção de biolubrificante em escala de laboratório
}

\author{
Contribution of inputs to total cost of the bioprocess for biolubricant \\ production on a laboratory scale
}

\author{
Milson dos Santos Barbosa ${ }^{1}$ (i) https://orcid.org/0000-0001-5412-7865 \\ Afonso Aurélio de Carvalho Peres ${ }^{2}$ (i) https://orcid.org/0000-0001-9037-0715 \\ Álvaro Silva Lima1 (i) https://orcid.org/0000-0002-0603-8187 \\ Cleide Mara Faria Soares ${ }^{1}$ (i) https://orcid.org/0000-0002-5932-9842
}

\begin{abstract}
Resumo
Os biolubrificantes são alternativas ecologicamente corretas aos óleos lubrificantes minerais, especialmente por serem biodegradáveis. Com base nisso, pesquisadores e empresários têm direcionado os seus esforços para desenvolver e implementar processos eficientes e sustentáveis sem onerar o financeiro das indústrias de lubrificantes. Para esse fim, é crucial avaliar os insumos que mais contribuem para a composição do custo operacional de produção, uma vez que a partir então será possível implementar uma planta piloto comercialmente. Neste cenário, o presente estudo avalia a representatividade dos itens de produção que compõem o custo operacional de um bioprocesso de produção de biolubrificantes, a partir da hidroesterificação enzimática do óleo de Moringa oleifera Lam, em escala de laboratório. Os resultados revelaram que dentre todos os itens, o biocatalisador (lipase Candida rugosa) apresentou maior participação na composição dos custos, representando cerca de $31 \%$ dos custos operacionais de produção do bioprocesso. A análise indicou que a substituição da lipase Candida rugosa pela lipase Eversa ${ }^{\circledR}$ Transform 2.0 promoveria uma redução substancial de cerca de $26 \%$ nos custos operacionais. Outrossim, o escalonamento da produção, a certificação de sustentabilidade e os incentivos fiscais que podem ser associados a implementação industrial do bioprocesso desenvolvido podem promover a redução dos custos operacionais de produção do biolubrificante.

Palavras-chave: Biolubrificante. Bioprocesso. Certificação verde. Custo operacional.
\end{abstract}

\begin{abstract}
Biolubricants are environmentally friendly alternatives to mineral lubricating oils, especially as they are biodegradable. Based on this, researchers and entrepreneurs have directed their efforts to develop and implement efficient and sustainable processes without burdening the lubricant industry's finances. For this purpose, it is crucial to evaluate the inputs that most contribute to the composition of the operating cost of production, since from then on it will be possible to implement a pilot plant commercially. In this scenario, the present study evaluates the representativeness of the inputs in the composition of the operational cost of a biolubricant production bioprocess, based on the enzymatic hydroesterification of Moringa oleifera Lam oil, on a laboratory scale. The results revealed that among all the inputs, the biocatalyst (Candida rugosa lipase) had greater participation in the composition of costs, making up around $31 \%$ of the operational costs of production of the bioprocess. The analysis indicated that replacing Candida rugosa lipase with lipase Eversa ${ }^{\circledR}$ Transform 2.0 would provide a substantial reduction of approximately $26 \%$ in operating costs. Furthermore, the scaling of production, sustainability certification, and tax incentives that can be associated with the industrial implementation of the developed bioprocess can significantly reduce the operating costs of production of the biolubricant.
\end{abstract}

Keywords: Biolubricant. Bioprocess. Green certification. Operational cost.

\footnotetext{
${ }^{1}$ Universidade Tiradentes, Programa de Pós-Graduação em Engenharia de Processos. Av. Murilo Dantas, 300, 49032-971, Aracaju, SE, Brasil. Correspondência para/Correspondence to: C. M. F. SOARES. E-mail: cleide18@yahoo.com.br.

${ }^{2}$ Universidade Federal Fluminense, Programa de Pós-Graduação em Tecnologia Ambiental.
} 


\section{Introdução}

Os lubrificantes são usados em uma ampla gama de equipamentos, desde máquinas pesadas, como motores industriais, até equipamentos menores, como discos rígidos de computadores. No entanto, estima-se que mais de $50 \%$ de todos os lubrificantes usados em todo o mundo entram no ambiente devido à vazamentos, descarte inadequado e acidentes (Syahir et al., 2017; Singh et al., 2018). Como mais de 95\% dos lubrificantes que entram no meio ambiente são derivados do petróleo, esses contaminam o ar, o solo, a água e, consequentemente, afetam a vida animal e humana. Esse contexto incentivou o interesse em desenvolver e usar lubrificantes biodegradáveis, os chamados biolubrificantes (Reeves; Siddaiah; Menezes, 2017; Siddaiah et al., 2019).

As políticas de desenvolvimento de produtos com foco na sustentabilidade exigem a integração entre as questões ambientais, econômicas e sociais que abrangem toda a cadeia produtiva de indústrias. Na era da alta consciência ecológica e da busca de uma economia circular é imprescindível a síntese de biolubrificantes a partir de processos ambientalmente amigáveis com reaproveitamento dos resíduos gerados (Heikal et al., 2017; Singh et al., 2018). Outra questão importante é a viabilidade econômico-financeira dos processos verdes em progresso, especialmente em países emergentes, como o Brasil, que passa por um período de transição para um modelo de desenvolvimento mais sustentável (Costa et al., 2018). Em geral, o custo desses processos alternativos é afetado por insumos específicos que possuem baixa oferta e alta demanda. Assim, as estimativas de despesas de produção são cruciais e devem ser realizadas preferencialmente nos estágios iniciais de um projeto, mesmo quando estiverem disponíveis apenas dados experimentais, fornecendo informações necessárias para orientar o desenvolvimento das atividades de pesquisa em escala de laboratório.

Com o intuito de clarificar essa questão e com base no estudo recente de (Barbosa et al., 2021) que revelou dados promissores para o bioprocesso de valorização integral de sementes de Moringa oleifera Lam e produção de biolubrificante, a partir da hidroesterificação enzimática do óleo de Moringa oleifera Lam foi analisada a representatividade dos itens que compõem o custo operacional de produção do biolubrificante, em escala de laboratório. A tecnologia proposta combina o reaproveitamento de biomassa residual oriunda da extração do óleo de Moringa oleifera Lam com a produção de um biolubrificante que apresenta propriedades específicas aquelas inerentes aos fluidos hidráulicos da classe ISO VG 68. Por se tratar de um processo de produção em escala de laboratório, a identificação dos custos envolvidos nos processos pode contribuir em estudos futuros referentes a análise de investimento para a indústria de lubrificantes. O intuito é identificar a participação dos itens envolvidos no processo e a representatividade destes na composição do custo operacional de produção do bioprocesso e, assim, permitir que ações sejam realizadas visando a redução dos custos futuros da implementação do bioprocesso em escala piloto e industrial. Portanto, a abordagem adotada avalia os custos operacionais por categoria dos itens de produção necessários para a produção 
do biolubrificante, em laboratório. Além disso, visando a ampliação a uma escala industrial da tecnologia desenvolvida, são apresentados os incentivos fiscais que podem ser associados a uma unidade de biorrefinaria para valorização integral de sementes de Moringa oleifera Lam e a produção de biolubrificante.

\section{Materiais e Métodos}

O diagrama de fluxo do processo de valorização integral de sementes de Moringa oleifera Lam e produção de biolubrificante em escala de laboratório (Figura 1), engloba cinco processos principais, desde a extração do óleo das sementes de Moringa oleifera Lam até a produção do biolubrificante, com base no estudo promissor de Barbosa et al. (2021). No processo inicial para extração do óleo de Moringa oleifera Lam as sementes de Moringa oleifera Lam são trituradas em um moinho de facas e seguem para uma peneira para atingir um tamanho de partícula inferior a 60 mesh. Então, as sementes seguem para um extrator Soxhlet a $68^{\circ} \mathrm{C}$ utilizando hexano (solvente) por 480 min, realizando assim a extração do óleo. Além do óleo vegetal rico em ácido oleico, a unidade de extração fornece dois subprodutos: a fração residual sólida (biomassa lignocelulósica), utilizada para a fabricação do suporte para imobilização do biocatalisador; e o hexano, que é recuperado e reutilizado após a separação do óleo em um evaporador rotativo.

A fração residual proveniente da extração do óleo de Moringa oleifera Lam segue para o processo de fabricação do suporte para imobilização enzimática. Inicialmente, a fração lignocelulósica é transferida para uma estufa e, após a evaporação das moléculas remanescentes de hexano, o material é triturado e peneirado para atingir um tamanho de partícula entre 120 e 140 mesh. Na sequência, essa fração residual é utilizada juntamente com tetraetilortossilicato, ácido clorídrico, água destilada, hidróxido de amônio e etanol para a preparação da matriz híbrida via técnica sol-gel em um sistema inerte de nitrogênio mantido a $35^{\circ} \mathrm{C}$ por $155 \mathrm{~min}$. Então, o material é transferido para um refrigerador que após $1.440 \mathrm{~min}$ segue para um sistema de filtração com hexano. Por fim, o suporte híbrido é levado a um dessecador e, após $4.320 \mathrm{~min}$, é peneirado para obtenção de partículas de tamanho semelhante (32-60 mesh).

No processo de fabricação do biocatalisador imobilizado, o suporte híbrido, o hexano e uma solução enzimática (preparada com a lipase Candida rugosa (LCR) e água destilada) são adicionados em um reator de vidro disposto em um sistema com agitação orbital a 200 rpm, deixado por $195 \mathrm{~min}$ a $25^{\circ} \mathrm{C}$. A mistura é refrigerada por $1.440 \mathrm{~min}$ e, então, segue para o sistema de filtração sob vácuo para remoção das partículas de proteína que não foram suficientemente fixadas no suporte. Enfim, o biocatalisador imobilizado é mantido em um dessecador por 2.880 min e armazenado em refrigerador até ser utilizado. 


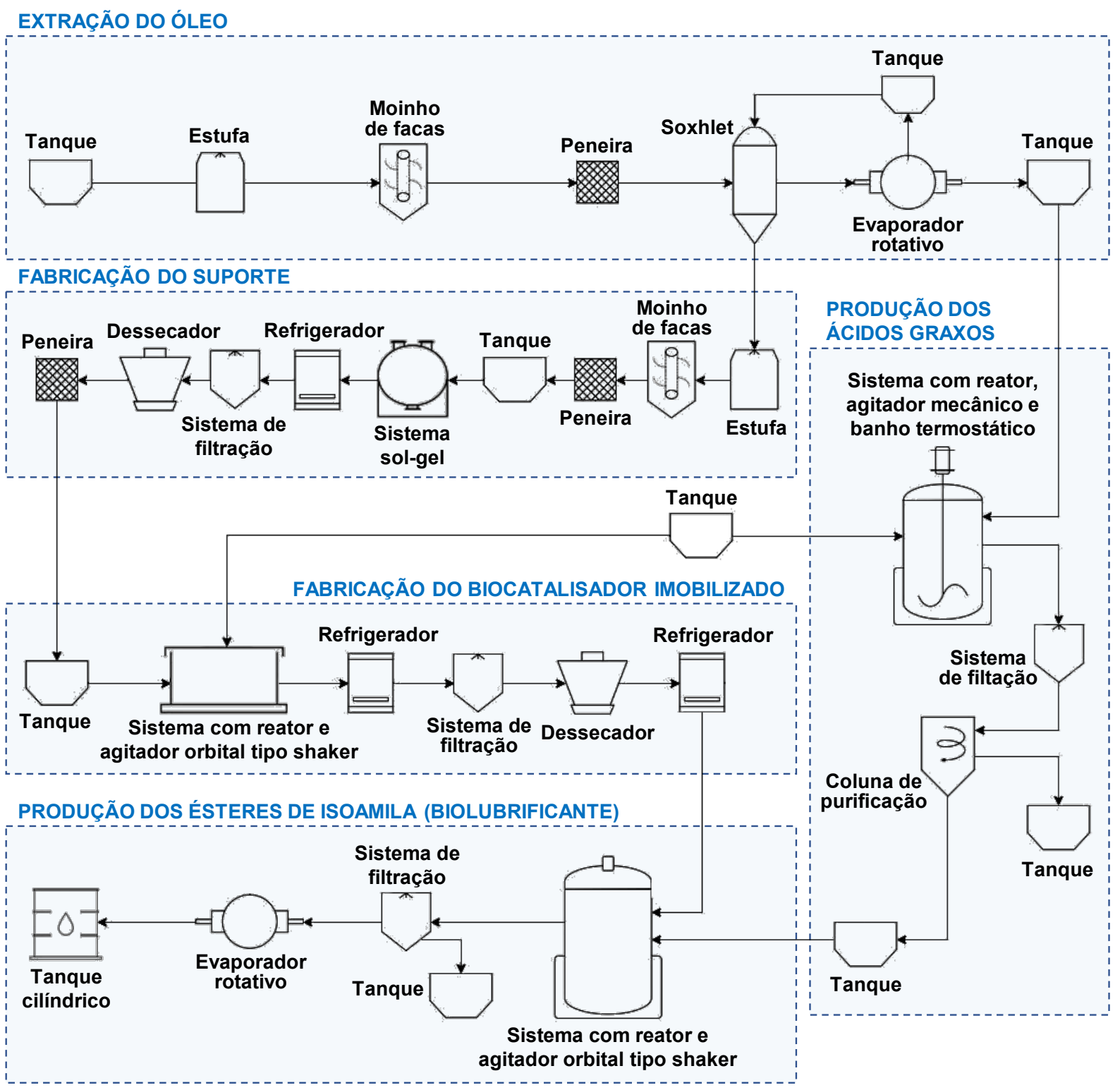

Figura 1 - Diagrama de fluxo do bioprocesso para valorização integral de sementes de Moringa oleifera Lam e produção de biolubrificante em escala de laboratório.

Fonte: Elaborada pelos autores (2021).

O processo de hidrólise enzimática para a produção dos ácidos graxos é iniciado quando o óleo bruto de Moringa oleifera Lam, a água destilada e a LCR são inseridos em um reator de vidro operando com impelidor do tipo hélice, sob agitação mecânica a 1.000 rpm, condicionado a uma temperatura de $37^{\circ} \mathrm{C}$ por $60 \mathrm{~min}$. Os produtos da reação de hidrólise seguem para um funil de decantação, em que a fase orgânica é recuperada e degomada com água destilada fervente. $\mathrm{Na}$ sequência, a quantidade remanescente de água é removida dos ácidos graxos com a passagem do material por coluna de filtração preenchida com sulfato de sódio anidro e lã de vidro. No processo final de produção dos ésteres de isoamila com propriedades lubrificantes, os ácidos graxos purificados são adicionados juntamente com o álcool isoamílico no reator de vidro acoplado em um banho maria incubador com agitação orbital a 200rpm. Após a homogeneização do meio reacional, o biocatalisador imobilizado é incorporado na mistura. A reação de esterificação é mantida a uma temperatura controlada de $40^{\circ} \mathrm{C}$ por 60 min e, após (c) (i) 
esse período, o biocatalisador imobilizado é recuperado por filtração com funil de Buchner. Os produtos da reação são neutralizados com a adição de uma solução de carbonato de sódio e, em seguida, os ésteres são separados por meio de lavagens com água destilada fervente. $A$ mistura segue para um evaporador rotativo e, no final, é disposta na presença de peneiras moleculares para remoção de água.

A contribuição dos itens de produção utilizados para a produção do biolubrificante em laboratório foi calculado com base no seu consumo em diferentes etapas do bioprocesso. Os insumos foram classificados e enquadrados em categorias, a saber: materiais, reagentes químicos, máquinas, equipamentos e energia. Os preços de todos os itens foram coletados no mercado, considerando como mês e ano de referência, o mês de março do ano de 2021, a partir de informações obtidas junto aos produtores e/ou fornecedores brasileiros e podem sofrer reajustes devido à demanda ou às diferenças cambiais em outros países. Custos de energia elétrica $(\mathrm{R} \$ \mathrm{kWh}$ ) foram estimados com base nas tarifas de energia elétrica do fornecedor local (Energisa), considerando a modalidade tarifária convencional de classe industrial. Por se tratar de uma análise em escala de laboratório, o custo operacional de produção não inclui despesas como impostos, subsídios, mão de obra humana, análise da qualidade, manutenção e arrendamento de área. Os dados coletados foram transcritos em planilha eletrônica e os custos foram estimados usando as funções estatísticas do MS-Exce ${ }^{\circledR}$.

\section{Resultados e Discussão}

Todos os itens envolvidos na produção do biolubrificante em laboratório foram enquadrados em cinco categorias: materiais, reagentes químicos, máquinas, equipamentos e energia. Na classe dos materiais foram incluídos os itens de produção, como: a enzima utilizada (LCR) e as sementes de Moringa oleifera Lam, enquanto entre os reagentes químicos estão o hexano, o tetraetilortossilicato e o álcool isoamílico. O extrator Soxhlet e os sistemas com agitador mecânico ou orbital foram classificados como maquinários, ao passo que as peneiras, o dessecador e as vidrarias em geral foram incluídos na classe dos equipamentos. Em energia, mensurou-se o consumo de energia elétrica para realização de todo o processo na produção do biolubrificante. As representatividades por categoria dos itens na composição do custo operacional de produção do biolubrificante em laboratório são apresentadas na Figura 2.

Os materiais são o principal contribuinte para o custo de produção do bioprocesso quando comparado a outros itens, representando aproximadamente $52 \%$ do custo operacional da produção do biolubrificante em escala de laboratório (Figura 2). Entre as demais categorias que geram custos para o bioprocesso estão aqueles que foram despendidos com energia elétrica $(\approx 21 \%)$, equipamentos $(\approx 16 \%)$, reagentes químicos $(\approx 6 \%)$ e as máquinas $(\approx 5 \%)$. É notório que o maior custo unitário entre todos os itens está associado ao biocatalisador, uma vez que $60 \%$ dos custos entre todos os materiais são decorrentes dos gastos com a LCR. Contudo, é importante ressaltar que a LCR imobilizada em suporte híbrido (sílica com biomassa residual 
da extração do óleo das sementes de Moringa oleifera Lam) reteve sua atividade original após oito ciclos sob as condições reacionais de esterificação considerada no estudo. Assim, o biocatalisador imobilizado pode ser recuperado e reinserido ao reator de esterificação por pelo menos oito lotes de produção do biolubrificante, o que poderia contribuir para uma redução de cerca de $24 \%$ dos custos inerentes a aquisição dos materiais.

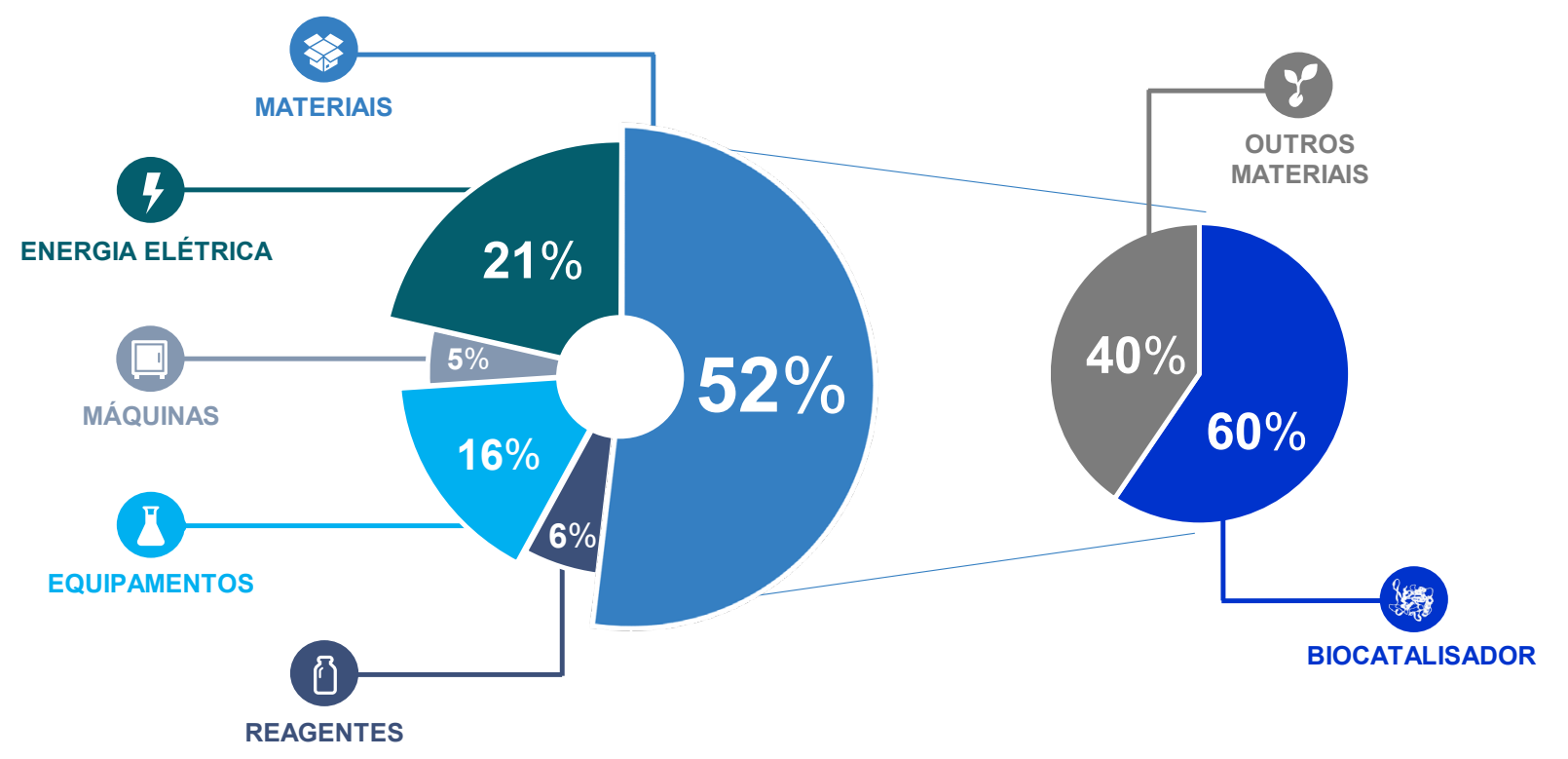

Figura 2 - Contribuição por categoria dos itens envolvidos no custo de produção do biolubrificante em escala de laboratório.

Fonte: Elaborada pelos autores (2021).

A substituição da LCR por uma lipase de baixo custo pode ser alternativa para redução dos custos operacionais de produção do bioprocesso. Neste sentido, a lipase Eversa ${ }^{\circledR}$ Transform $^{2}$ 2.0, tem sido relatada recentemente como uma formulação enzimática líquida de baixo custo (Martínez-Sanchez et al., 2020). Derivada do Aspergillus oryzae geneticamente modificado, a lipase foi introduzida no mercado pela Novozymes no ano de 2016 como um biocatalisador mais econômico e competitivo com a finalidade de converter glicerídeos e ácidos graxos livres em biodiesel. Estudos demonstraram desempenhos satisfatórios da lipase imobilizada em diferentes suportes durante a síntese enzimática de ésteres etílicos ou metílicos (Remonatto et al., 2018; Miranda et al., 2020; Monteiro et al., 2021).

Além da produção de biodiesel, foi reportado recentemente o potencial da lipase Eversa ${ }^{\circledR}$ Transform 2.0 imobilizada pela técnica de agregados enzimáticos reticulados (CLEA) para a síntese de biolubrificantes por transesterificação de óleo de cozinha residual com diferentes álcoois (metanol, etanol, octano e isoamílico) (Guimarães et al., 2021). O estudo revelou que o biocatalisador imobilizado (Eversa-mCLEA) demonstrou maior especificidade para álcoois de cadeia longa (octano- $\mathrm{C}_{8} \mathrm{H}_{18}$ ) e ramificados (isoamílico - $\mathrm{C}_{5} \mathrm{H}_{12} \mathrm{O}$ ) em comparação com os álcoois de cadeia curta (metanol- $\mathrm{CH}_{4} \mathrm{O}$ e etanol- $\mathrm{C}_{2} \mathrm{H}_{6} \mathrm{O}$ ), apresentando melhor desempenho catalítico durante a síntese de ésteres isoamílicos com um rendimento de éster em torno de 90\% (em 
peso) após $72 \mathrm{~h}$ de reação. Esses resultados sugerem que a substituição da LCR pela lipase Eversa $^{\circledR}$ Transform 2.0 poderia manter a eficiência do bioprocesso considerado. Assim, a substituição da lipase foi considerada na análise dos custos operacionais de produção, em laboratório. Os dados estimados indicam que substituir a LCR pela lipase Eversa ${ }^{\circledR}$ Transform 2.0 geraria uma redução de cerca de $26 \%$ nos custos operacionais de produção do biolubrificante.

\section{Incentivos fiscais associados à uma biorrefinaria para produção de biolubrificante}

A Figura 3 apresenta a perspectiva esquemática que ilustra uma unidade de bioprocesso para valorização integral de sementes de Moringa oleifera Lam e produção de biolubrificante (Barbosa et al., 2021). Com vistas à certificação verde e almejando uma economia circular, essa estratégia prevê o uso do óleo bruto de Moringa oleifera Lam como fonte de ácido oleico (74,5\%). Além disso, o resíduo sólido da extração de óleo de Moringa oleifera Lam foi utilizado para produzir um suporte híbrido para imobilização de lipase, em dois processos enzimáticos. No primeiro processo, a produção de ácidos graxos livres a partir do óleo de Moringa oleifera Lam ocorre por hidrólise enzimática do completa sob condições otimizadas de reação $\left(37^{\circ} \mathrm{C}\right.$, razão mássica óleo:água a $25 \%$, atividade hidrolítica de $550 \mathrm{U} / g_{\text {óleo }}$ e agitação mecânica a 1.000rpm, $60 \mathrm{~min}$ ). A dispensa de controle do $\mathrm{pH}$ e ausência de emulsificantes comerciais no meio reacional reduz substancialmente os custos de produção associados à purificação dos produtos obtidos.

No segundo processo biocatalítico, a síntese de ésteres ocorre via esterificação direta de ácidos graxos do óleo bruto de Moringa oleifera Lam com álcool isoamílico em um sistema isento de solventes, com conversão máxima de $94 \%$ após 60 min de reação a $40^{\circ} \mathrm{C}, 1: 1$ de razão molar

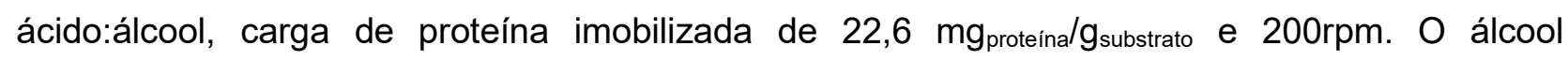
isoamílico foi selecionado por ser o álcool em maior concentração no óleo fúsel, subproduto gerado durante a produção de bioetanol, portanto, o seu uso atende as demandas de biorrefinaria. Ademais, a isenção de solvente e o uso de uma proporção equimolar de ácido para álcool eliminam etapas de processamento, reduzem o desperdício e os riscos e, portanto, viabilizam um processo de produção mais eficiente, econômico e sustentável. Nesta reação, também é possível reutilizar o biocatalisador imobilizado no suporte híbrido por pelo menos oito ciclos sucessivos sem qualquer redução significativa na sua produtividade. Além disso, os ésteres isoamílicos apresentaram boas propriedades lubrificantes para atender às especificações impostas aos fluidos hidráulicos da classe ISO VG 68 (Barbosa et al., 2021). 


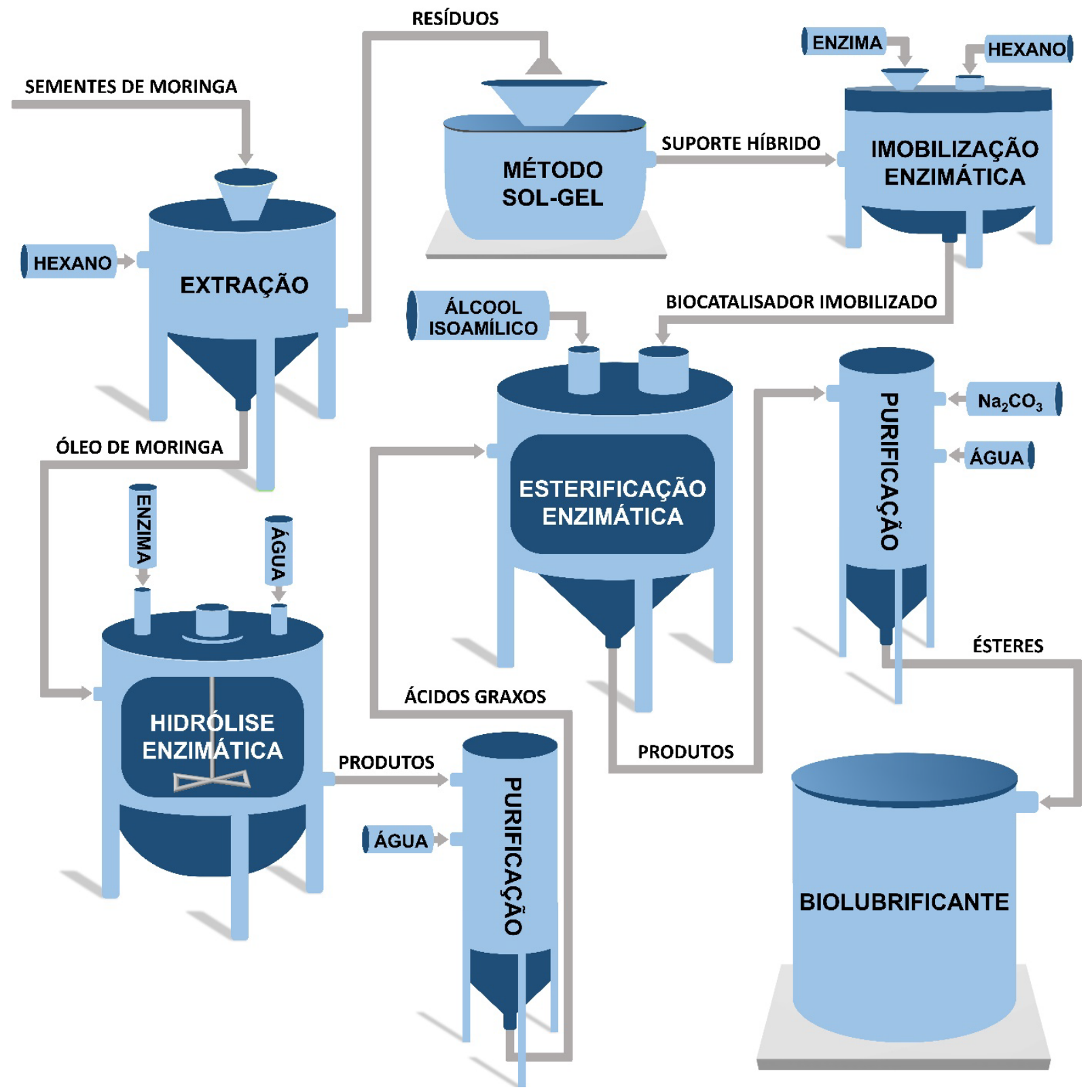

Figura 3 - Diagrama esquemático da unidade de bioprocesso integrado para a produção de biolubrificante.

Fonte: Elaborado pelos autores (2021).

Dentre as principais ações que garantem a certificação verde e geram redução de impostos para as indústrias estão a uso de fontes renováveis, a menor geração de resíduos e o desenvolvimento de produtos menos agressivos à natureza. Essas são práticas intrínsecas a tecnologia desenvolvida, a qual projeta uma biorrefinaria para produção de biolubrificante em favor da economia circular. Assim, após a implementação do bioprocesso em escala industrial, algumas das isenções ou descontos que podem ser aplicados sobre cargas tributárias, tais como Imposto Predial e Territorial Urbano (IPTU), Imposto sobre Circulação de Mercadorias e Serviços (ICMS), Imposto sobre Produtos Industrializados (IPI), Imposto Sobre Serviços (ISS), Imposto de Renda (IR), e sobre o Imposto de Importação (II). O IPTU Verde é um dos incentivos fiscais verdes mais difundidos e, a depender do município, concede descontos entre $5 \%$ e $20 \%$ sobre o 
imposto de propriedades urbanas para empresas ou indústrias com certificação sustentável que adotam medidas de proteção, preservação e recuperação do meio ambiente (Gramkow e AngerKraavi, 2018).

\section{Conclusão}

O bioprocesso proposto tem vantagens substanciais sobre os processos tradicionais de produção de lubrificantes, em termos de materiais de partida, geração de resíduos e consumo energético. A análise apresentada indica que a substituição do biocatalisador por uma enzima de baixo custo comercial reduziria substancialmente o custo operacional de produção do bioprocesso, o que pode contribuir para a implementação em escala industrial. Embora a avaliação ambiental e uma análise econômica mais crítica sejam necessárias, um enorme avanço foi dado para produção sustentável de lubrificantes biodegradáveis. Portanto, o estudo desenvolvido abre novos caminhos para ampliar a escala de laboratório para escala piloto e, eventualmente, para escala industrial, podendo reduzir substancialmente os custos operacionais de produção do biolubrificante.

\section{Agradecimentos}

Este estudo foi financiado pela Coordenação de Aperfeiçoamento de Pessoal de Nível Superior (CAPES, código financeiro 001), Conselho Nacional de Desenvolvimento Científico e Tecnológico (CNPq) e Fundação de Apoio à Pesquisa e à Inovação Tecnológica do Estado de Sergipe (FAPITEC/SE).

\section{Colaboradores}

M. S. BARBOSA colaborou com a concepção, planejamento, coleta e análise de dados, além da elaboração do manuscrito. A. A. C. PERES colaborou com a análise e interpretação de dados, além da revisão do manuscrito. A. S. LIMA colaborou com a concepção e revisão do manuscrito e C. M. F. SOARES colaborou com a concepção, planejamento e revisão do manuscrito.

\section{Referências}

Barbosa, M. S. et al. Biolubricant production under zero-waste Moringa oleifera Lam biorefinery approach for boosting circular economy. Industrial Crops \& Products, v. 167, n. 1, p. 113542, 2021.

Costa, J. A. F. et al. Energy law and regulation in Brazil. [S.I.]: Springer, 2018.

Gramkow, C.; Anger-Kraavi, A. Could fiscal policies induce green innovation in developing countries? The case of Brazilian manufacturing sectors. Climate Policy, v. 18, n. 2, p. 246-257, 2018.

Guimarães, J. R. et al. Immobilization of Eversa ${ }^{\circledR}$ transform via CLEA technology using waste cooking oil. Molecules, v. 23, n. 1, p. 193, 2021.

Heikal, E. K. et al. Manufacturing of environment friendly biolubricants from vegetable oils. 
Egyptian Journal of Petroleum, v. 26, n. 1, p. 53-59, 2017.

Martínez-Sanchez, J. A. et al. Immobilized biocatalysts of Eversa ${ }^{\circledR}$ Transform 2.0 and lipase from Thermomyces lanuginosus: Comparison of some properties and performance in biodiesel production. Catalysts, v. 10, n. 7, p. 1-19, 2020.

Miranda, L. P. et al. Composites of crosslinked aggregates of Eversa ${ }^{\circledR}$ transform and magnetic nanoparticles: performance in the ethanolysis of soybean oil. Catalysts, v. 10, n. 8, p. 9-11, 2020.

Monteiro, R. R. C. et al. Liquid lipase preparations designed for industrial production of biodiesel. Is it really an optimal solution? Renewable Energy, v. 164, p. 1566-1587, 2021.

Reeves, C. J.; Siddaiah, A.; Menezes, P. L. A review on the science and technology of natural and synthetic biolubricants. Journal of Bio- and Tribo-Corrosion, v. 3, n. 1, p. 11, 2017.

Remonatto, D. et al. Production of FAME and FAEE via alcoholysis of sunflower oil by Eversa lipases immobilized on hydrophobic supports. Applied Biochemistry and Biotechnology, v. 185, n. 3, p. 705-716, 2018.

Siddaiah, A. et al. Influence of environmental friendly multiphase lubricants on the friction and transfer layer formation during sliding against textured surfaces. Journal of Cleaner Production, v. 209, n. 3, p. 1245-1251, 2019.

Singh, Y. et al. Sustainability of a non-edible vegetable oil based bio-lubricant for automotive applications: a review. Process Safety and Environmental Protection, v. 111, n. 1, p. 701-713, 2018.

Syahir, A. Z. et al. A review on bio-based lubricants and their applications. Journal of Cleaner Production, v. 168, n. 1, p. 997-1016, 2017.

Como citar este artigo/How to cite this article

BARBOSA, M. S. et al. Contribuição dos insumos no custo total do bioprocesso para produção de biolubrificante em escala de laboratório. Sustentabilidade: Diálogos Interdisciplinares, v. 2, e215519, 2021. https://doi.org/10.24220/2675-7885v2e2021a5519

Recebido em 6 de dezembro de 2021 e aprovado em 14 de dezembro de 2021. 